Revista de Matemática: Teoría y ApliCACiones 2017 24(1) : 9-28

CIMPA - UCR ISSN: 1409-2433 (PRINT), 2215-3373 (ONLINE)

\title{
LÍMITES DIRECTOS DE PROLONGACIONES DE ALGEBROIDES DE LIE
}

\section{DIRECT LIMITS OF LIE ALGEBROIDS \\ PROLONGATIONS}

\author{
PATRICK CABAU* \\ 23/Jun/2015; Revised: 18/Aug/2016; \\ Accepted: 7/Oct/2016
}

Revista de Matemática: Teoría y Aplicaciones is licensed under a Creative Commons
Reconocimiento-NoComercial-Compartirigual 4.0 International License.
Creado a partir de la obra en http://www.revistas.ucr.ac.cr/index.php/matematica

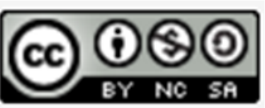

${ }^{*}$ Lycée Pierre de Fermat, Parvis des Jacobins, 31000 Toulouse, France. E-Mail: Patrick.Cabau@ac-toulouse.fr 


\title{
Resumen
}

Probamos que se puede definir estructuras de espacios convenientes sobre límites directos de algebroides de Lie y sus prolongaciones.

Palabras clave: límite directo; algebroide de Lie; prolongación de algebroide de Lie; cálculo diferencial conveniente.

\begin{abstract}
We prove that direct limits of Lie algebroids and their prolongations can be endowed with structures of convenient spaces.
\end{abstract}

Keywords: direct limit; Lie algebroid; prolongation of Lie algebroid; convenient calculus.

Mathematics Subject Classification: 46A13, 46T05, 58A32

\section{Introduction}

Los algebroides de Lie definidos por J. Pradines en [17] constituyen una generalización natural de las álgebras de Lie y de los fibrados tangentes a una variedad.

En los últimos años, esta noción se ha revelado fecunda en Mecánica, Geometría simpléctica y teoría del Control óptimo. En [19], Weinstein plantea el problema de un posible desarrollo de un formalismo geométrico sobre algebroides de Lie similar al formalismo de Klein en Mecánica Lagrangiana. En [15], Martínez da una respuesta positiva a este problema usando la noción de prolongación de un algebroide de Lie introducida por Higgins y Mackenzie en [10].

En este artículo nos interesamos a los límites directos de tales estructuras y obtenemos los resultados dados en los teoremas 21 y 22 de la última sección: se puede definir sobre estos límites una estructura conveniente.

En la sección 2 recordamos el formalismo conveniente desarrollado en [12]. Desarrollamos la noción de límite directo de diferentes estructuras (espacios vectoriales topologicos, variedades, fibrados vectoriales) en las secciones 3 y 4 . Entonces, obtenemos estructuras convenientes sobre estos límites (Proposición 6 y Proposición 11). En la sección 5 recordamos las nociones de algebroides de Lie y sus prolongaciones. En la última sección probamos que se puede definir sobre límites directos de tales objetos estructuras convenientes y damos el ejemplo del oscilador armónico conveniente. 


\section{Cálculo diferencial conveniente}

Con el fin de equipar un espacio vectorial topológico de Haussdorf localmente convexo (e.v.t.l.c.) $E$ con una estructura diferencial, como introducida por Frölicher, Kriegl y Michor, se utiliza la noción de curva diferenciable $c: \mathbb{R} \rightarrow E$ de clase $C^{\infty}$ que no plantea ningún problema.

La propiedad clave es la de $c^{\infty}$-completitud.

Definición 1 Se dice que un e.v.t.l.c. $E$ es $c^{\infty}$-completo o conveniente si se cumple la propiedad siguiente: Dado $B \subset E$ un conjunto cerrado, acotado y absolutamente convexo, el espacio lineal $E_{B}$ generado por $B$ es un espacio de Banach.

Un e.v.t.l.c. conveniente es Mackey completo (cf. [12] Teorema 2.14).

La $c^{\infty}$-topología de un e.v.t.l.c. es la topología final inducida por la familia de las curvas $C^{\infty} \mathbb{R} \rightarrow E$; se denotará por $c^{\infty} E$. A sus conjuntos abiertos los llamaremos $c^{\infty}$-abiertos.

Nótese que la $c^{\infty}$-topología es en general más fina que la topología original. En los espacios de Fréchet, esta topología coincide con la topología del e.v.t.l.c.

En general, $c^{\infty} E$ no es un espacio vectorial localmente convexo.

Sean $E$ y $F$ dos espacios convenientes y sea $U \subset E$ un $c^{\infty}$-abierto. Se dice que una aplicación $f: E \supset U \rightarrow F$ es $c^{\infty}$ si $f \circ c \in C^{\infty}(\mathbb{R}, F)$ para cada $c \in C^{\infty}(\mathbb{R}, U)$.

Además, se puede definir una estructura de espacio vectorial $c^{\infty}$-completo sobre el espacio $C^{\infty}(U, F)$ (cf. [12], 2.3 (5)).

Proposición 2 Los límites, las sumas directas y los límites directos estrictos de espacios convenientes son espacios convenientes.

Las nociones de variedad conveniente (véase [12], 27.) y de fibrado vectorial conveniente (véase [12], 29.) se definen de manera natural.

\section{Límites directos de espacios vectoriales topológicos}

En esta sección vamos a referirnos a [1], [6] y [7].

Sea $(I, \leq)$ un conjunto direccionado. Un sistema directo en una categoría $\mathbb{A}$ es un par $\mathcal{S}=\left(X_{i}, \varepsilon_{i}^{j}\right)_{i \in I, j \in I, i \leq j}$ donde $X_{i}$ es un objeto de la categoría y $\operatorname{los} \varepsilon_{i}^{j}: X_{i} \longrightarrow X_{j}$ son homomorfismos (bonding maps) que cumplen las propiedades siguientes: 
1. $\forall i \in I, \varepsilon_{i}^{i}=\operatorname{Id}_{X_{i}}$.

2. $\forall(i, j, k) \in I^{3}, i \leq j \leq k, \varepsilon_{j}^{k} \circ \varepsilon_{i}^{j}=\varepsilon_{i}^{k}$.

Un cono sobre $\mathcal{S}$ es un $\operatorname{par}\left(X, \varepsilon_{i}\right)_{i \in I}$ donde $X \in \mathrm{ob} \mathbb{A}, \varepsilon_{i}: X_{i} \longrightarrow X$ y los homomorfismos $\varepsilon_{i}^{j}: X_{i} \longrightarrow X_{j}$ verifican las relaciones $\varepsilon_{j} \circ \varepsilon_{i}^{j}=\varepsilon_{i}$ para $i \leq j$.

Un cono $\left(X, \varepsilon_{i}\right)_{i \in I}$ es un límite directo de $\mathcal{S}$ si para cualquier cono $\left(Y, \theta_{i}\right)_{i \in I}$ sobre $\mathcal{S}$ existe un único homomorfismo $\psi: X \longrightarrow Y$ tal que $\psi \circ \varepsilon_{i}=\theta_{i}$. En tal

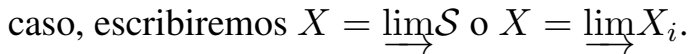

Cuando $I=\mathbb{N}$ dotado de la relación de orden en los números naturales, los sistemas directos numerables se llaman sucesiones directas.

\subsection{Límites directos de conjuntos}

Sea $\mathcal{S}=\left(X_{i}, \varepsilon_{i}^{j}\right)_{i \in I, j \in I, i \leq j}$ un sistema directo de conjuntos $(\mathbb{A}=\mathbb{S E T})$.

Sea $\mathcal{U}=\coprod_{i \in I} X_{i}=\left\{(x, i): x \in X_{i}\right\}$ la unión disjunta de los conjuntos $X_{i}$ con la inclusión canónica $\begin{array}{cccc}\iota_{i}: & X_{i} & \longrightarrow & \mathcal{U} \\ x & \mapsto & (x, i) .\end{array}$ equivalencia $\sim$ sobre $\mathcal{U}$ de la manera siguiente: $\iota_{i}(x) \sim \iota_{j}(y)$ si existe $k \in I$ $: i \leq k$ y $j \leq k$ tales que $\varepsilon_{i}^{k}(x)=\varepsilon_{j}^{k}(y)$. Tenemos el conjunto cociente $X=\mathcal{U} / \sim$ y la aplicación $\varepsilon_{i}: \pi \circ \iota_{i}$ donde $\pi: \mathcal{U} \longrightarrow \mathcal{U} / \sim$ es la aplicación canónica suprayectiva.

Entonces, $\left(X, \varepsilon_{i}\right)$ es el límite directo $\mathcal{S}$ en la categoría $\mathbb{S E T}$.

Si cada $\varepsilon_{i}^{j}$ es inyectiva entonces $\varepsilon_{i}$ es inyectiva. Por supuesto $S$ es equivalente al sistema directo de los subconjuntos $\varepsilon_{i}\left(X_{i}\right) \subset X$, con las inclusiones canónicas.

\subsection{Límites directos de espacios topólogicos}

Si $\mathcal{S}=\left(X_{i}, \varepsilon_{i}^{j}\right)_{i \in I, j \in I, i \leq j}$ es un sistema directo de espacios topológicos $X_{i}$ donde $\varepsilon_{i}^{j}$ son aplicaciones continuas, entonces el límite directo $\left(X, \varepsilon_{i}\right)_{i \in I}$ en la categoría de conjuntos coincide con el límite directo en la categoría $\mathbb{T} \mathbb{O P}$ de los espacios topológicos si $X$ tiene la $D L$-topología, i.e. la topología más fina para la cual todas las aplicaciones $\varepsilon_{i}$ son continuas. Entonces $O \subset X$ es abierto si y sólo si $\varepsilon_{i}^{-1}(O)$ es abierto en $X_{i}$ para cada $i \in I$.

$\mathcal{S}$ es estricto si cada $\varepsilon_{i}^{j}$ es un encaje. En esta situación, cada $\varepsilon_{i}$ es un encaje.

Demos ahora algunas propiedades de sucesiones crecientes de espacios topológicos ([7], Lema 1.7): 
Proposición 3 Sea $X_{1} \subset X_{2} \subset \cdots$ una sucesión creciente de espacios topológicos tales que las inclusiones son continuas. Ponemos en $X=\bigcup_{n \in \mathbb{N}^{*}} X_{n}$ la topología final correspondiente a la familia de las inclusiones $\varepsilon_{n}: X_{n} \hookrightarrow X$ (i.e. la DL-topología). Entonces, tenemos las propiedades siguientes:

1. Si cada $X_{n}$ es $T_{1}$, entonces $X$ es $T_{1}$.

2. Si $O_{n} \subset X_{n}$ es abierto y $O_{1} \subset O_{2} \subset \cdots$, entonces $O=\bigcup_{n \in \mathbb{N}^{*}} O_{n}$ es un abierto de $X$ y la DL-topología sobre $O=\lim _{n} O_{n}$ coincide con la topología inducida por $X$.

3. Si cada $X_{n}$ es localmente compacto, entonces $X$ es Haussdorf.

4. Si cada $X_{n}$ es $T_{1}$ y $K \subset X$ es compacto, entonces $K \subset X_{n}$ para cierto $n$.

\subsection{Límites directos de espacios vectoriales topológicos de dimension finita}

Sea $E$ un espacio vectorial real de dimensión numerable.

Sea $E_{1} \subset E_{2} \subset \cdots$ una sucesión creciente de subespacios vectoriales de $E$ de dimensión finita tal que $E=\bigcup_{n \in \mathbb{N}^{*}} E_{n}$. Se obtiene una sucesión directa estricta de subespacios vectoriales dotada de la topología final por las inclusiones $E_{n} \hookrightarrow E$.

Entonces $O \subset X$ es abierto si y sólo si $\varepsilon_{n}^{-1}(O)$ es un abierto de $X_{n}$ para cada $n \in \mathbb{N}$.

Puesto que los espacios son de dimensiones finitas esta topología coincide con la más fina topología de espacio vectorial localmente convexo ([6], Ejemplo 3.5); el conjunto de todos los subconjuntos equilibrados, absorbentes y convexos es una base para esta topología.

Además $E$ es un espacio vectorial conveniente ([6], Lema 6.1).

Tenemos la relación que sigue entre la diferenciabilidad $C^{\infty}$ y la $c^{\infty}$ ([7], Lema 1.9)

Lema 4 Para una aplicación $f: O \longrightarrow F$ donde $O=\bigcup_{n \in \mathbb{N}^{*}} O_{n}$ es una sucesión creciente de conjuntos abiertos $\left(O_{n} \subset E_{n}\right)$ y $F$ un e.v.t.l.c. real y Mackey completo tenemos la equivalencia siguiente: $f$ es $C^{\infty}$ si y sólo si $f$ es $c^{\infty}$.

Ejemplo 5 El espacio vectorial $\mathbb{R}^{\infty}$, también denotado por $\mathbb{R}^{(\mathbb{N})}$, de todas las sucesiones finitas es el límite directo estricto de $\left(\mathbb{R}^{i}, \varepsilon_{i}^{j}\right)_{(i, j) \in \mathbb{N}^{2}, i \leq j}$ donde 
$\varepsilon_{i}^{j}:\left(x_{1}, \ldots, x_{i}\right) \mapsto\left(x_{1}, \ldots, x_{i}, 0, \ldots, 0\right)$.

Es un espacio vectorial numerable y conveniente ([12], 47.1). Una base de $\mathbb{R}^{\infty}$ es $\left(e_{i}\right)_{i \in \mathbb{N}^{*}}$ donde $e_{i}=\left(0, \ldots, 0, \underset{i^{\text {th }}}{1 \text { term }}, 0, \ldots\right) \in \varepsilon_{i}\left(\mathbb{R}^{i}\right)$.

\section{Límites directos de variedades}

\subsection{Límites directos de sucesiones crecientes de variedades de dimensión finita}

Combinando los resultados obtenidos por Glöckner ([7], Teorema 1 y Proposición 3.6), obtenemos:

Teorema 6 Sea $\mathcal{M}=\left(M_{i}, \varepsilon_{i}^{j}\right)_{i \in \mathbb{N}^{*}, j \in \mathbb{N}^{*}, i \leq j}$ una sucesión directa de variedades de clase $C^{\infty}$ paracompactas de dimensión finita donde $\varepsilon_{i}^{j}: M_{i} \longrightarrow M_{j}$ son inmersiones inyectivas de clase $C^{\infty}$. Sea $s=\sup _{i \in \mathbb{N}^{*}}\left(\operatorname{dim} M_{i}\right) \in \mathbb{N} \cup\{+\infty\}$. Hay una única estructura de variedad $c^{\infty}$ modelada sobre $\mathbb{R}^{\infty}$ por la cual $\varepsilon_{n}: M_{n} \longrightarrow M=\bigcup_{i \in \mathbb{N}^{*}} M_{i}$ es una aplicación de clase $c^{\infty}$ para cada $n \in \mathbb{N}^{*} y$ tal que $\left(M, \varepsilon_{n}\right)_{n \in \mathbb{N}^{*}}=\lim _{\longrightarrow} \mathcal{S}$ en la categoría de las variedades convenientes.

Ejemplo 7 La esfera $\mathbb{S}^{\infty}$ ([12], 47.2).- El espacio conveniente $\mathbb{R}^{\infty}$ es equipado con el producto escalar débil dado por la suma finita $\langle x, y\rangle=\sum_{i} x_{i} y_{i}$ bilineal y acotada. El límite inductivo de $\mathbb{S}^{1} \subset \mathbb{S}^{2} \subset \cdots$ es el subconjunto cerrado $\mathbb{S}^{\infty}=\left\{x \in \mathbb{R}^{\infty}:\langle x, x\rangle=1\right\}$ de $\mathbb{R}^{\infty}$. Es una variedad conveniente modelada sobre $\mathbb{R}^{\infty}$.

\subsection{Funciones sobre límites directos de variedades}

Sea $\mathcal{M}=\left(M_{i}, \varepsilon_{i}^{j}\right)_{i \in \mathbb{N}^{*}, j \in \mathbb{N}^{*}, i \leq j}$ una sucesión directa de variedades de clase $C^{\infty}$ donde $\varepsilon_{i}^{j}: M_{i} \longrightarrow M_{j}$ son inmersiones inyectivas $C^{\infty}$. Podemos identificar $M_{i}$ con el subconjunto $\varepsilon_{i}^{j}\left(M_{i}\right)$ de $M_{j}$. El álgebra de las funciones reales definidas sobre $M_{i}$ se denotará por $\mathcal{F}\left(M_{i}\right)$. Entonces, podemos definir la sucesión $\mathcal{F}=\left(\mathcal{F}\left(M_{i}\right), \delta_{i}^{j}\right)_{i \in \mathbb{N}^{*}, j \in \mathbb{N}^{*}, i \leq j}$ con las aplicaciones

$$
\begin{aligned}
& \delta_{i}^{j}: \mathcal{F}\left(M_{j}\right) \longrightarrow \mathcal{F}\left(M_{i}\right) \\
& f_{j} \quad \longmapsto \quad f_{i}
\end{aligned}
$$


donde $\delta_{i}^{j}=\left(\varepsilon_{i}^{j}\right)^{*}$, i.e. $\delta_{i}^{j}\left(f_{j}\right)=f_{j} \circ \varepsilon_{i}^{j}$. Estas aplicaciones satisfacen las condiciones $\delta_{i}^{j} \circ \delta_{j}^{k}=\delta_{i}^{k}$ para cada $i \leq j \leq k$. Entonces $\mathcal{F}$ es una sucesión proyectiva y se puede identificar el límite proyectivo $\varliminf_{\longleftarrow} \mathcal{F}\left(M_{i}\right) \operatorname{con} \bigcap_{i=1}^{+\infty} \mathcal{F}\left(M_{i}\right)$.

Sea $f=\lim f_{i}$ el límite proyectivo de funciones $f_{i}: M_{i} \longrightarrow \mathbb{R}$ de clase

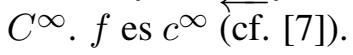

Para cada $x \in M$ se define la diferencial $d f_{x}: T_{x} M \longrightarrow \mathbb{R}$ de la manera siguiente: si $x \in M_{k}$, entonces $d f(x)=d f_{k}\left(x_{k}\right): T_{x_{k}} M_{k} \longrightarrow \mathbb{R}$.

\subsection{Límites directos de campos de vectores}

Sea $\mathcal{M}=\left(M_{i}, \varepsilon_{i}^{j}\right)_{i \in \mathbb{N}^{*}, j \in \mathbb{N}^{*}, i \leq j}$ una sucesión directa de variedades paracompactas de clase $C^{\infty}$ donde $\varepsilon_{i}^{j}: M_{i} \longrightarrow M_{j}$ son inmersiones inyectivas $C^{\infty}$. Entonces podemos equipar $\mathcal{T}=\left(T M_{i}, T \varepsilon_{i}^{j}\right)_{i \in \mathbb{N}^{*}, j \in \mathbb{N}^{*}, i \leq j}$ con una estructura de fibrado vectorial conveniente (cf. [18]).

Sea $\left(X_{i}\right)_{i \in \mathbb{N}^{*}}$ una sucesión de campos de vectores $X_{i} \in \mathfrak{X}\left(M_{i}\right)$ tal que:

$$
T \varepsilon_{i}^{j} \circ X_{i}=X_{j} \circ \varepsilon_{i}^{j}
$$

Podemos definir una sección $c^{\infty}$ de $\underset{\longrightarrow}{\lim } T M_{i}$ como $\underset{\longrightarrow}{\lim } X_{i}$.

Ejemplo $8 X=\sum_{i=1}^{+\infty} x_{i} \frac{\partial}{\partial x_{i}}$ es un campo de vectores de $\mathbb{R}^{\infty}$ definido de la manera siguiente: sea $x \in \mathbb{R}^{\infty}$; existe $n=n(x) \in \mathbb{N}^{*}$ tal que $x=\iota_{n}\left(x_{1}, \ldots, x_{n}\right)$ donde $\iota_{n}: \mathbb{R}^{n} \hookrightarrow \mathbb{R}^{\infty}$ es la inyección canónica. Tenemos así $X_{x}=\sum_{i=1}^{n(x)} x_{i} \frac{\partial}{\partial x_{i}}$.

\subsection{Límites directos de grupos de Lie}

Podemos encontrar en [7], Teorema 4.3 (d), el resultado siguiente a propósito del límite directo de una sucesión de groupos de Lie de dimension finita.

Proposición 9 Sea una sucesión de grupos de Lie reales de dimension finita y de clase $C^{\infty} \mathcal{G}=\left(G_{i}, \varepsilon_{i}^{j}\right)_{i \in \mathbb{N}^{*}, j \in \mathbb{N}^{*}, i \leq j}$ donde $\varepsilon_{i}^{j}: G_{i} \longrightarrow G_{j}$ son $C^{\infty}$. homomorfismos. Entonces $G=\underline{\longrightarrow} \lim _{i}$ es un grupo de Lie $c^{\infty}$-regular.

Ejemplo $10 G L(\infty, \mathbb{R})=\underset{\lim }{\longrightarrow} G L\left(\mathbb{R}^{n}\right)$ es un grupo de Lie conveniente. 


\subsection{Límites directos de fibrados vectoriales}

Denotamos la inyección canónica $\mathbb{R}^{i} \hookrightarrow \mathbb{R}^{j}$ por $\iota_{i}^{j}$.

Sea $\mathcal{M}=\left(M_{i}, \varepsilon_{i}^{j}\right)_{i \in \mathbb{N}^{*}, j \in \mathbb{N}^{*}, i \leq j}$ una sucesión directa de variedades paracompactas de clase $C^{\infty}$ y de dimension finita $\left(\operatorname{dim} M_{i}=d_{i}\right)$ donde $\varepsilon_{i}^{j}: M_{i} \longrightarrow$ $M_{j}$ son inmersiones inyectivas de clase $C^{\infty}$. Podemos suponer que $M_{1} \subset M_{2} \subset$ … Aquí consideramos la situación donde $\sup d_{i}=+\infty$.

Par cada número natural $i$, sea $\left(E_{i}, \pi_{i}, M_{i}\right)$ un fibrado vectorial cuya fibra es isomorfa al espacio vectorial $\mathbb{E}_{i}$ de dimensión $r_{i}$ (que se puede identificar a $\mathbb{R}^{r_{i}}$ ) donde $\sup _{i \in \mathbb{N}^{*}} r_{i}=+\infty$. Supongamos que $\mathcal{E}=\left(E_{i}, \lambda_{i}^{j}\right)_{i \in \mathbb{N}^{*}, j \in \mathbb{N}^{*}, i \leq j}$ es una sucesión directa de variedades donde $\lambda_{i}^{j}: E_{i} \longrightarrow E_{j}$ son inmersiones inyectivas de clase $C^{\infty}$ y morfismos de fibrados vectoriales. Supongamos también que $E_{1} \subset E_{2} \subset \ldots$

A la sucesión $\mathcal{E}=\left(\left(E_{i}, \pi_{i}, M_{i}\right)_{i}, \lambda_{i}^{j}\right)_{i \in \mathbb{N}^{*}, j \in \mathbb{N}^{*}, i \leq j}$ la llamaremos limite directo de fibrados vectoriales si, para cada $x_{i} \in M_{i}$, existe un sistema directo de trivializaciones $\left(U_{i}, \Psi_{i}\right)\left(\operatorname{con} U_{1} \subset U_{2} \subset \cdots\right.$ y $U_{i}$ abierto de $\left.M_{i}\right)$ de $\left(E_{i}, \pi_{i}, M_{i}\right)$ donde $\Psi_{i}: \pi_{i}^{-1}\left(U_{i}\right) \longrightarrow U_{i} \times \mathbb{R}^{r_{i}}$ son difeomorfismos locales tales que $x_{i} \in U_{i}$ y para cada par $(i, j) \in \mathbb{N}^{2}, i \leq j$, tenemos las condiciones de compatibilidad:

$$
\left(\varepsilon_{i}^{j} \times \iota_{r_{i}}^{r_{j}}\right) \circ \Psi_{i}=\Psi_{j} \circ \lambda_{i}^{j} .
$$

La proposición siguiente generaliza el resultado de [18] a propósito de límite directo de fibrados tangentes.

Proposición 11 Sea $\mathcal{E}=\left(\left(E_{i}, \pi_{i}, M_{i}\right)_{i}, \lambda_{i}^{j}\right)_{i \in \mathbb{N}^{*}, j \in \mathbb{N}^{*}, i \leq j}$ una sucesión directa de fibrados vectoriales. Entonces $\left(\underset{\longrightarrow}{\lim E_{i}}, \underset{\longrightarrow}{\lim } \pi_{i}, \underset{\longrightarrow}{\lim } M_{i}\right)$ puede ser equipado con una estructura de fibrado vectorial conveniente cuya base es modelada sobre $\mathbb{R}^{\infty}$ y cuyo grupo estructural es el grupo de Lie conveniente $G L(\infty, \mathbb{R})=$ $\lim _{\longrightarrow} G L\left(\mathbb{R}^{n}\right)$.

Demostración. Definamos en primer lugar una estructura de variedad sobre $E=\underline{\lim } E_{i}$.

Para cada $i \in \mathbb{N}^{*}, E_{i}$ es un espacio topológico localmente compacto; entonces, $\lim _{\longrightarrow} E_{i}$ es Hausdorff (cf. [9]).

Sea $\vec{v} \in E$; definamos une carta en $v$ como sigue. Puesto que $v$ pertenece a $\underset{\lim }{\longrightarrow} E_{i}$, existe $n \in \mathbb{N}^{*}$ tal que $v=\lambda_{n}\left(v_{n}\right)$ con $v_{n} \in E_{n}$ y $\pi_{n}\left(v_{n}\right)=x \in M_{n}$. Para $i \geq n$, la trivialización local $\Psi_{i}: \pi_{i}^{-1}\left(U_{i}\right) \longrightarrow U_{i} \times \mathbb{R}^{r_{i}}$ da origen, via 
una carta $\varphi_{i}: U_{i} \longrightarrow \mathbb{R}^{d_{i}}$, a una carta $\psi_{i}: \pi_{i}^{-1}\left(U_{i}\right) \longrightarrow \mathbb{R}^{d_{i}} \times \mathbb{R}^{r_{i}}$. Puesto que $\lambda_{i}^{j}$ es un morfismo sobre $\varepsilon_{i}^{j}$, tenemos $\pi_{j} \circ \lambda_{i}^{j}=\varepsilon_{i}^{j} \circ \pi_{i}$ y podemos definir $\pi=\lim _{i}: \underline{\lim } E_{i} \longrightarrow \underline{\lim } M_{i}$ por $\pi(v)=\pi_{n}\left(v_{n}\right)=x \in M_{n} \subset \underline{\lim } M_{i}$. Tenemos entonces $\stackrel{\lim }{\longrightarrow}\left(\pi_{i}\right)^{-1}\left(U_{i}\right)=\pi^{-1}\left(\stackrel{\lim }{\longrightarrow} U_{i}\right)$.

Puesto que el diagrama siguiente es conmutativo

$$
\begin{array}{cccc}
\left(\pi_{i}\right)^{-1}\left(U_{i}\right) & \lambda_{i}^{j} & \left(\pi_{j}\right)^{-1}\left(U_{j}\right) \\
\Psi_{i} \downarrow & & \downarrow \Psi_{j} \\
U_{i} \times \mathbb{R}^{r_{i}} & \stackrel{\left(\varepsilon_{i}^{j} \times \iota_{r_{i}}^{r_{j}}\right)}{\longrightarrow} & U_{j} \times \mathbb{R}^{r_{j}} \\
&
\end{array}
$$

podemos definir una trivialización local del fibrado $\left(\underset{\longrightarrow}{\lim } E_{i}, \underset{\longrightarrow}{\lim } \pi_{i}, \lim _{\longrightarrow} M_{i}\right)$

$$
\begin{aligned}
& \Psi: \pi^{-1}\left(\lim _{i} U_{i}\right) \quad \longrightarrow \quad \stackrel{\lim U_{i}}{\longrightarrow} \times \mathbb{R}^{\infty} \\
& v=\lim _{i} v_{i} \mapsto \quad\left(x=\lim _{\longrightarrow} x_{i}, \widehat{v}=\lim _{\longrightarrow} \widehat{v}_{i}\right)
\end{aligned}
$$

donde $\widehat{v}_{i}=\theta_{\iota}\left(v_{i}\right) \operatorname{con} \theta_{\iota}=\operatorname{pr}_{2} \circ \Psi_{i}$.

$\Psi$ es un homeomorfismo como límite directo de homeomorfismos.

Utilizando las cartas $\left(U_{i}, \varphi_{i}\right)_{i \geq n}$ se puede definir una carta de $E$ en $v$ :

$$
\begin{aligned}
\psi: \pi^{-1}\left(\underline{\left.\lim U_{i}\right)}\right. & \longrightarrow \quad \mathbb{R}^{\infty} \times \mathbb{R}^{\infty} \\
v=\varliminf_{\lim } v_{i} & \mapsto \quad\left(\bar{x}=\underline{\lim } \overline{x_{i}}, \widehat{v}=\underline{\lim } \widehat{v_{i}}\right)
\end{aligned}
$$

donde $\overline{x_{i}}=\varphi_{i}\left(x_{i}\right)$.

Además $\theta_{x \mid \pi^{-1}(y)}=\lim _{\longrightarrow}\left(\theta_{i}\right)_{x_{i} \mid \pi_{i}^{-1}\left(y_{i}\right)}: \pi^{-1}(y) \longrightarrow\{y\} \times \mathbb{R}^{\infty}$ es lineal.

Probemos ahora que los cambios de cartas son $c^{\infty}$-difeomorfismos.

Consideramos dos cartas $\left(\pi^{-1}\left(U^{\alpha}\right), \psi^{\alpha}\right)$ y $\left(\pi^{-1}\left(U^{\beta}\right), \psi^{\beta}\right)$ en $v \in E$. Tenemos entonces:

$$
\tau^{\beta \alpha}=\psi^{\beta} \circ\left(\psi^{\alpha}\right)^{-1}: \psi^{\alpha}\left(\pi^{-1}\left(U^{\alpha} \cap U^{\beta}\right)\right) \longrightarrow \psi^{\beta}\left(\pi^{-1}\left(U^{\alpha} \cap U^{\beta}\right)\right)
$$

donde $\tau^{\alpha \beta} \circ\left(\iota_{d_{i}}, \iota_{r_{i}}\right)=\left(\iota_{d_{i}}, \iota_{r_{i}}\right) \circ \tau_{i}^{\alpha \beta}\left(\iota_{k}: \mathbb{R}^{k} \hookrightarrow \mathbb{R}^{\infty}\right.$ es la inyección canónica). Se sigue que $\tau^{\beta \alpha}=\left(\varphi^{\beta} \circ\left(\varphi^{\alpha}\right)^{-1}, \theta^{\beta} \circ\left(\theta^{\alpha}\right)^{-1}\right) \in \operatorname{Diff}^{\infty}\left(\mathbb{R}^{\infty}\right) \times G L(\mathbb{R}, \infty)$ donde $G l(\mathbb{R}, \infty)$ es un grupo de Lie conveniente ([12], 47.7).

Probemos ahora la $c^{\infty}$-diferenciabilidad de la proyección $\pi: E \longrightarrow M$. Sea $c$ : ]$-\varepsilon, \varepsilon\left[\longrightarrow \pi^{-1}(U) \in E\right.$ una curva tale que $c(0)=v$ donde $\pi(v)=x \in U \subset$ $M$. Puesto que $c(]-\varepsilon, \varepsilon[)$ es relativamente compacto, tenemos $c(]-\varepsilon, \varepsilon[) \subset$ $\left(\pi_{n}\right)^{-1}\left(U_{n}\right)\left(3,4\right.$.). Entonces $\pi \circ c=\pi_{n} \circ c_{n} \in C^{\infty}(]-\varepsilon, \varepsilon\left[, \mathbb{R}^{d_{n}}\right)$. Deducimos de esto que $\pi$ es $c^{\infty}$. 


\section{Algebroides de Lie}

Recordamos definiciones y propiedades utilizadas en [3] (ver también [11] y [16]).

\subsection{Definiciones. Expresión local. Ejemplos}

Sea $\tau: E \rightarrow M$ un fibrado vectorial sobre une variedad de dimensión finita cuya fibra es un espacio vectorial $\mathbb{E}$ de dimensión finita.

Un morfismo de fibrados vectoriales $\rho: E \rightarrow T M$ se llama un ancla. Este morfismo da origen a un $\mathcal{F}$-modulo $\rho: \Gamma(E) \rightarrow \Gamma(T M)=\mathfrak{X}(M)$ definido para cada $x \in M$ y cada sección $s$ de $E$ por: $(\underline{\rho}(s))(x)=\rho(s(x))$ y denotado también por $\rho$.

Llamaremos a $(E, \tau, M, \rho)$ un fibrado anclado.

Definición 12 Un casi-corchete sobre un fibrado anclado $(E, \tau, M, \rho)$ es un corchete $[.,]_{\rho}$ que satisface una regla de Leibniz:

$$
\forall f \in \mathcal{F}, \forall s_{1}, s_{2} \in \Gamma(E), \quad\left[s_{1}, f s_{2}\right]_{\rho}=f\left[s_{1}, s_{2}\right]_{\rho}+\left(\rho\left(s_{1}\right)\right)(f) s_{2} .
$$

Un fibrado anclado $(E, \tau, M, \rho)$ equipado con un casi-corchete es llamado casialgebroide de Lie.

Definición 13 Un corchete de Lie sobre un fibrado anclado $(E, \tau, M, \rho)$ es un casi-corchete que verifica la identidad de Jacobi:

$$
\forall s_{1}, s_{2}, s_{3} \in \Gamma(E),\left[s_{1},\left[s_{2}, s_{3}\right]_{\rho}\right]_{\rho}+\left[s_{2},\left[s_{3}, s_{1}\right]_{\rho}\right]_{\rho}+\left[s_{3},\left[s_{1}, s_{2}\right]_{\rho}\right]_{\rho}=0 .
$$

Un fibrado anclado $(E, \pi, M, \rho)$ equipado con un corchete de Lie se llama algebroide de Lie.

Cuando $(E, \tau, M, \rho)$ es un algebroide de Lie el corchete $[., .]_{\rho}$ es un morfismo de álgebras de Lie.

Ejemplo 14 Cualquier álgebra de Lie de dimensión finita es un algebroide de Lie sobre un punto.

Ejemplo 15 El fibrado tangente $\pi_{M}: T M \longrightarrow M$ a una variedad diferencial es un algebroide de Lie donde el ancla es $\mathrm{Id}_{T M}$ y el corchete de secciones es el corchete de Lie de los campos de vectores. 
Ejemplo $16 E=T M$ y $\rho=N$ es un tensor de Nijenhuis, i.e. un tensor que verifica la condición

$$
[N X, N Y]=N([N X, Y]+[X, N Y]-N([X, Y])) .
$$

$(T M, \pi, M, N)$ es un algebroide de Lie (cf. [2]) donde el ancla es $N$ y el corchete de secciones es el corchete $[., .]_{N}$ definido por:

$$
[X, Y]_{N}=[N X, Y]+[X, N Y]-N([X, Y]) .
$$

Ejemplo 17 Sea $(M, \Lambda)$ una variedad de Poisson. El fibrado cotangente $T^{*} M$ a una variedad diferencial está dotado de una estructura de algebroide de Lie donde el ancla es el morfismo $\Lambda^{\sharp}$ y el corchete en las 1-formas (cf. [14]) viene dado por:

$$
[\alpha, \beta]_{\Lambda}=L_{\Lambda^{\sharp} \beta}(\alpha)-L_{\Lambda^{\sharp} \alpha}(\beta)+d\left\langle\beta, \Lambda^{\sharp} \alpha\right\rangle
$$

$\operatorname{para} \alpha, \beta \in \Omega^{1}(M)$.

Si fijamos un sistema de coordenadas locales $\left(x^{i}\right)_{1 \leq i \leq n}$ en la base $M$ y una base local $\left(e_{\alpha}\right)_{1 \leq \alpha \leq m}$ de secciones de $\tau$, obtenemos un sistema de coordenadas locales $\left(x^{i}, y^{\alpha}\right)$ de $E$.

Tenemos una expresión local del ancla y del corchete:

$$
\rho\left(e_{\alpha}\right)=\rho_{\alpha}^{i} \frac{\partial}{\partial x^{i}} \mathrm{y}\left[e_{\alpha}, e_{\beta}\right]_{\rho}=C_{\alpha \beta}^{\gamma} e_{\gamma}
$$

donde las funciones $\rho_{\alpha}^{i}$ y $C_{\alpha \beta}^{\gamma}$ verifican relaciones debidas a la condición de compatibilidad y a la identidad de Jacobi.

\subsection{Cálculo en algebroides de Lie}

Dado un algebroide de Lie $(E, \tau, M, \rho)$ se definen la derivada de Lie y la diferencial exterior.

Derivada de Lie. Sea $s$ una sección de $E$. Se define la derivada de Lie $L_{s}^{\rho}$ - de una función $f \in \Omega^{0}(M, E)=\mathcal{F}$ de clase $C^{\infty}$ por:

$$
L_{s}^{\rho}(f)=L_{\rho \circ s}(f)=i_{\rho \circ s}(d f) .
$$

- de una $q$-forma $\omega \in \Omega^{q}(M, E)$ (donde $q>0$ ) por

$$
\begin{aligned}
\left(L_{s}^{\rho} \omega\right)\left(s_{1}, \ldots, s_{q}\right) & =L_{s}^{\rho}\left(\omega\left(s_{1}, \ldots, s_{q}\right)\right) \\
& -\sum_{i=1}^{q} \omega\left(s_{1}, \ldots, s_{i-1},\left[s, s_{i}\right]_{\rho}, s_{i+1}, \ldots, s_{q}\right) .
\end{aligned}
$$


Diferencial exterior. Se define la diferencial exterior $d_{\rho}$ - de una función $f \in \mathcal{F}$ por

$$
d_{\rho} f=t_{\rho} \circ d f .
$$

- de una $q$-forma $\omega \in \Omega^{q}(M, E)$ (donde $q>0$ ) por

$$
\begin{aligned}
\left(d_{\rho} \omega\right)\left(s_{0}, \ldots, s_{q}\right) & =\sum_{i=0}^{q}(-1)^{i} L_{s_{i}}^{\rho}\left(\omega\left(s_{0}, \ldots, \widehat{s}_{i}, \ldots, s_{q}\right)\right) \\
& +\sum_{0 \leq i<j \leq q}^{q}(-1)^{i+j}\left(\omega\left(\left[s_{i}, s_{j}\right]_{\rho}, s_{0}, \ldots, \widehat{s_{i}}, \ldots, \widehat{s_{j}}, \ldots, s_{q}\right)\right) .
\end{aligned}
$$

Si $\left(x^{i}\right)_{1 \leq i \leq n}$ son coordenadas locales sobre $M$ y si $\left\{e_{\alpha}\right\}_{1 \leq \alpha \leq m}$ es una base local de secciones de $\tau$ tenemos las expresiones locales siguientes:

$$
d x^{i}=\rho_{\alpha}^{i} e^{\alpha} \mathrm{y} d e^{\gamma}=-\frac{1}{2} C_{\alpha \beta}^{\gamma} e^{\alpha} \wedge e^{\beta}
$$

donde $\left(e^{\alpha}\right)$ es la base dual de $\left(e_{\alpha}\right)$.

Tenemos

$$
d_{\rho} \circ d_{\rho}=0 .
$$

La cohomología asociada con $d_{\rho}$ se denomina la cohomología del algebroide de Lie $(E, \tau, M, \rho)$.

\subsection{Morfismo de algebroides de Lie}

Definición 18 Un morfismo de fibrados vectoriales $\psi: E \rightarrow E^{\prime}$ sobre $f$ : $M \rightarrow M^{\prime}$ es un morfismo de algebroides de Lie $(E, \tau, M, \rho)$ y $\left(E^{\prime}, \tau^{\prime}, M^{\prime}, \rho^{\prime}\right)$ si $\psi^{*}: \Omega^{q}\left(M, E^{\prime}\right) \rightarrow \Omega^{q}(M, E)$ definida por:

$$
\left(\psi^{*} \alpha^{\prime}\right)_{x}\left(s_{1}, \ldots, s_{q}\right)=\alpha_{f(x)}^{\prime}\left(\psi \circ s_{1}, \ldots, \psi \circ s_{q}\right)
$$

verifica la relación:

$$
d_{\rho} \circ \psi^{*}=\psi^{*} \circ d_{\rho^{\prime}}
$$

\subsection{Prolongaciones de algebroides de Lie}

Vamos a recordar la definición de la estructura de algebroide de Lie sobre la prolongación de un algebroide de Lie mediante una fibración (véase [5], [16] y [4]). 
Sea $(E, \tau, M, \rho)$ un algebroide de Lie de rango $m$ sobre una variedad $M$ de dimensión $n$ y sea $\nu: P \longrightarrow M$ una fibración de rango $q$, esto es, una submersión sobreyectiva.

Para cada punto $p \in P$, consideramos el conjunto

$$
\mathcal{T}_{p}^{E} P=\left\{(b, v) \in E_{x} \times T_{p} P: \rho(b)=T_{p} \nu(v)\right\}
$$

donde $T \nu: T P \longrightarrow T M$ es la applicación tangente a $\nu$ y $\nu(p)=x \in M$.

El conjunto $\mathcal{T}^{E} P=\bigcup_{p \in P} \mathcal{T}_{p}^{E} P$ tiene una estructura natural de fibrado vectorial cuya proyección es $\tau_{P}^{E}:(b, v) \mapsto \nu(v)$.

Denotaremos de manera redundante $(b, v)$ por $(p, b, v)$.

El fibrado vectorial $\tau_{P}^{E}: \mathcal{T}^{E} P \rightarrow P$ admite una estructura de algebroide de Lie denominada prolongación del algebroide de Lie E mediante la fibración $\nu \mathrm{o}$ fibrado E-tangente a $P$.

El ancla $\rho_{P}: \mathcal{T}^{E} P \rightarrow T P$ es la proyección sobre el tercer factor, i.e. $\rho_{P}(p, b, v)=v$.

Con el fin de definir un corchete de secciones de $\tau_{P}^{E}$ vamos a considerar secciones particulares.

Una sección $Z \in \Gamma\left(\tau_{P}^{E}\right)$ se dice que es proyectable si existe una sección $\sigma$ de $\tau: E \longrightarrow M$ y un campo de vectores $U$ sobre $P$ proyectable mediante al campo de vectores $\rho(\sigma)$ y tales que $Z(p)=(p, \sigma(\nu(p)), U(p))$ para todo $p \in P$.

El corchete de dos secciones $Z_{1}$ y $Z_{2}$ dadas por $Z_{i}(p)=\left(p, \sigma_{i}(\nu(p)), U_{i}(p)\right)$, $i=1,2$, es:

$$
\left[Z_{1}, Z_{2}\right]_{\rho_{P}}(p)=\left(p,\left[\sigma_{1}, \sigma_{2}\right]_{\rho}(\nu(p)),\left[U_{1}, U_{2}\right](p)\right)
$$

para cada $p \in P$.

Es fácil probar que se puede elegir una base local de secciones proyectables del espacio $\Gamma\left(\tau_{P}^{E}\right)$.

Si $\left(x^{i}, u^{A}\right)_{1 \leq i \leq n, 1 \leq A \leq q}$ son coordenadas locales sobre $P$ y si $\left\{e_{\alpha}\right\}_{1 \leq \alpha \leq m}$ es una base local de secciones de $\tau: E \longrightarrow M$ podemos definir una base local $\left\{\mathcal{X}_{\alpha}, \mathcal{V}_{A}\right\}_{1 \leq \alpha \leq m, 1 \leq A \leq q}$ de secciones de $\tau_{P}^{E}$ dadas por:

$$
\mathcal{X}_{\alpha}(p)=\left(p, e_{\alpha}(\nu(p)), \rho_{\alpha}^{i}\left(\frac{\partial}{\partial x^{i}}\right)_{p}\right) \quad \text { y } \quad \mathcal{V}_{A}(p)=\left(p, 0,\left(\frac{\partial}{\partial u^{A}}\right)_{p}\right) .
$$

Si $z=(p, b, v)$ partenece a $\mathcal{T}^{E} P$ donde $b=z^{\alpha} e_{\alpha}$, entonces $v$ es de la forma

$$
v=\rho_{\alpha}^{i} z^{\alpha} \frac{\partial}{\partial x^{i}}+v^{A} \frac{\partial}{\partial u^{A}}
$$


y podemos escribir:

$$
z=z^{a} \mathcal{X}_{\alpha}(p)+v^{A} \mathcal{V}_{A}(p)
$$

Para $Z \in \Gamma\left(\tau_{P}^{E}\right)$ dada localmente por $Z=Z^{\alpha} \mathcal{X}_{\alpha}+V^{A} \mathcal{V}_{A}$ se tiene que

$$
\rho_{P}(Z)=\rho_{\alpha}^{i} Z^{\alpha} \frac{\partial}{\partial x^{i}}+V^{A} \frac{\partial}{\partial u^{A}}
$$

\subsection{Prolongación de morfismos de algebroides de Lie}

Sean $\nu: P \longrightarrow M$ y $\nu^{\prime}: P^{\prime} \longrightarrow M^{\prime}$ dos fibraciones. Sea $\Psi: P \longrightarrow P^{\prime}$ una aplicación fibrada sobre $\varphi: M \longrightarrow M^{\prime}$. Consideramos dos algebroides de Lie $\tau: E \longrightarrow M$ y $\tau^{\prime}: E^{\prime} \longrightarrow M^{\prime}$ y una aplicación $\Phi: E \longrightarrow E^{\prime}$ fibrada sobre $\varphi$. Si $\Phi$ es admisible podemos definir una aplicación admisible $T^{\Phi} \Psi: \mathcal{T}^{E} P \longrightarrow \mathcal{T}^{E^{\prime}} P^{\prime}$ por

$$
T^{\Phi} \Psi(p, b, v)=(\Psi(p), \Phi(b), T \Psi(v)) .
$$

Recordamos el resultado siguiente (véase [15]):

Proposición $19 T^{\Phi} \Psi$ es un morfismo de algebroides de Lie si y sólo si $\Phi$ es un morfismo de algebroides de Lie .

\section{Límites directos de prolongaciones de algebroides de Lie}

Definición 20 Se llama a $\left(E_{i}, \tau_{i}, M_{i}, \rho_{i}\right)_{i \in \mathbb{N}^{*}}$ sucesión directa de algebroides de Lie si

1. $\left(\left(E_{i}, \lambda_{i}^{j}\right)\right)_{i \in \mathbb{N}^{*}, j \in \mathbb{N}^{*}, i \leq j}$ es una sucesión directa de fibrados vectoriales de dimensiones finitas $\left(\tau_{i}: E_{i} \rightarrow M_{i}\right)$ sobre la sucesión directa de variedades de dimensiones finitas $\left(\left(M_{i}, \varepsilon_{i}^{j}\right)\right)_{i \in \mathbb{N}^{*}, j \in \mathbb{N}^{*}, i \leq j}$.

2. Para cada $i, j \in \mathbb{N}^{*}$ tales que $i \leq j$, tenemos

$$
\rho_{j} \circ \lambda_{i}^{j}=T \varepsilon_{i}^{j} \circ \rho_{i} .
$$

3. $\lambda_{i}^{j}: E_{i} \rightarrow E_{j}$ es un morfismo de los algebroides de Lie $\left(E_{i}, \tau_{i}, M_{i}, \rho_{i}\right)$ y $\left(E_{j}, \tau_{j}, M_{j}, \rho_{j}\right)$. 
Tenemos el resultado siguiente:

Teorema 21 Sea $\left(E_{i}, \tau_{i}, M_{i}, \rho_{i}\right)_{i \in \mathbb{N}^{*}}$ una sucesión directa de algebroides de Lie. Entonces $\left(\stackrel{\lim E_{i}}{,} \underset{\lim }{\longrightarrow}, \underset{\longrightarrow}{\longrightarrow} M_{i}, \lim _{\rightarrow}\right)$ es un algebroide de Lie conveniente.

\section{Demostración.}

1. $\left(\stackrel{\lim }{\longrightarrow} E_{i}, \underset{\longrightarrow}{\longrightarrow} \tau_{i}, \lim M_{i}\right)$ es un fibrado vectorial conveniente sobre la variedad conveniente $\underset{\lim }{\longrightarrow} M_{i}$ modelada sobre $\mathbb{R}^{\infty}$ (cf. Proposición 11).

2. Sean $\left(s_{i}^{1}\right)_{i \in \mathbb{N}^{*}}$ y $\left(s_{i}^{2}\right)_{i \in \mathbb{N}^{*}}$ directas sucesiones de secciones de fibrados vectoriales $\pi_{i}: E_{i} \rightarrow M_{i}$. Entonces se cumplen las condiciones

$$
\left\{\begin{array}{l}
\lambda_{i}^{j} \circ s_{i}^{1}=s_{j}^{1} \circ \varepsilon_{i}^{j} \\
\lambda_{i}^{j} \circ s_{i}^{2}=s_{j}^{2} \circ \varepsilon_{i}^{j}
\end{array}\right.
$$

Tenemos que probar la compatibilidad de los corchetes:

$$
\lambda_{i}^{j} \circ\left[s_{i}^{1}, s_{i}^{2}\right]_{E_{i}}=\left[s_{j}^{1}, s_{j}^{2}\right]_{E_{j}} \circ \varepsilon_{i}^{j}
$$

y de las propiedades de Leibniz:

$$
\lambda_{i}^{j} \circ\left[s_{i}^{1}, g_{i} \times s_{i}^{2}\right]_{E_{i}}=\left[s_{j}^{1}, g_{j} \times s_{j}^{2}\right]_{E_{j}} \circ \varepsilon_{i}^{j} .
$$

a) Con el fin de probar la compatibilidad de los corchetes utilizamos los morfis$\operatorname{mos} \lambda_{i}^{j}: E_{i} \longrightarrow E_{j}$ de algebroides de Lie sobre $\varepsilon_{i}^{j}: M_{i} \longrightarrow M_{j}$ que satisfacen:

$$
d_{\rho_{i}} \circ\left(\lambda_{i}^{j}\right)^{*}=\left(\lambda_{i}^{j}\right)^{*} \circ d_{\rho_{j}}
$$

y por tanto, aplicados a $\alpha_{j} \in \Omega^{1}\left(M_{j}, E_{j}\right)$ :

$$
\left(d_{\rho_{i}} \circ\left(\lambda_{i}^{j}\right)^{*}\left(\alpha_{j}\right)\right)\left(s_{i}^{1}, s_{i}^{2}\right)=\left(\left(\lambda_{i}^{j}\right)^{*} \circ d_{\rho_{j}}\left(\alpha_{j}\right)\right)\left(s_{i}^{1}, s_{i}^{2}\right) .
$$

El primer miembro es igual a

$$
\begin{aligned}
\left(d_{\rho_{i}} \circ\left(\lambda_{i}^{j}\right)^{*}\left(\alpha_{j}\right)\right)\left(s_{i}^{1}, s_{i}^{2}\right) \\
=L_{\rho_{i} \circ s_{i}^{1}}\left(\left(\left(\lambda_{i}^{j}\right)^{*}\left(\alpha_{j}\right)\right)\left(s_{i}^{2}\right)\right)-L_{\rho_{i} \circ s_{i}^{2}}\left(\left(\left(\lambda_{i}^{j}\right)^{*}\left(\alpha_{j}\right)\right)\left(s_{i}^{1}\right)\right) \\
\quad-\left(\left(\lambda_{i}^{j}\right)^{*}\left(\alpha_{j}\right)\right)\left[s_{i}^{1}, s_{i}^{2}\right]_{E_{i}} \\
=X_{j}^{1}\left(\alpha_{j}\left(\lambda_{i}^{j} \circ s_{i}^{2}\right)\right)-X_{j}^{2}\left(\alpha_{j}\left(\lambda_{i}^{j} \circ s_{j}^{1}\right)\right)-\alpha_{j}\left(\lambda_{i}^{j} \circ\left[s_{i}^{1}, s_{i}^{2}\right]_{E_{i}}\right) .
\end{aligned}
$$

donde $X_{j}^{a}=\rho_{j} \circ s_{j}^{a}$ con $a=1,2$ cumplen la relación: $X_{j}^{a}\left(f_{j}\right)=X_{i}^{a}\left(f_{i}\right)$ donde $f_{j}=\alpha_{j} \circ s_{j}$. 
El segundo miembro es igual a

$$
\begin{aligned}
& \left(\left(\lambda_{i}^{j}\right)^{*}\left(d_{\rho_{j}}\left(\alpha_{j}\right)\right)\right)\left(s_{i}^{1}, s_{i}^{2}\right) \\
& =d_{\rho_{j}}\left(\alpha_{j}\right)\left(\lambda_{i}^{j} \circ s_{i}^{1}, \lambda_{i}^{j} \circ s_{i}^{2}\right) \\
& =L_{\rho_{j} \circ \lambda_{i}^{j} \circ s_{i}^{1}}\left(\alpha_{j}\left(\lambda_{i}^{j} \circ s_{i}^{2}\right)\right)-L_{\rho_{j} \circ \lambda_{i}^{j} \circ s_{i}^{2}}\left(\alpha_{j}\left(\lambda_{i}^{j} \circ s_{i}^{1}\right)\right)-\alpha_{j}\left[\lambda_{i}^{j} \circ s_{i}^{1}, \lambda_{i}^{j} \circ s_{i}^{2}\right]_{E_{j}} \\
& =L_{\rho_{j} \circ s_{j}^{1}}\left(\alpha_{j}\left(\lambda_{i}^{j} \circ s_{i}^{2}\right)\right)-L_{\rho_{j} \circ s_{j}^{2}}\left(\alpha_{j}\left(\lambda_{i}^{j} \circ s_{i}^{1}\right)\right)-\alpha_{j}\left[\lambda_{i}^{j} \circ s_{i}^{1}, \lambda_{i}^{j} \circ s_{i}^{2}\right]_{E_{j}} \\
& =X_{j}^{1}\left(\alpha_{j}\left(\lambda_{i}^{j} \circ s_{i}^{2}\right)\right)-X_{j}^{2}\left(\alpha_{j}\left(\lambda_{i}^{j} \circ s_{j}^{1}\right)\right)-\alpha_{j}\left[\lambda_{i}^{j} \circ s_{i}^{1}, \lambda_{i}^{j} \circ s_{i}^{2}\right]_{E_{j}} .
\end{aligned}
$$

En particular, para cada $\alpha_{j} \in \Omega^{1}\left(M_{j}, E_{j}\right)$,

$\alpha_{j}\left(\lambda_{i}^{j}\left(\left[s_{i}^{1}, s_{i}^{2}\right]_{E_{i}}\right)\right)=\alpha_{j}\left[\lambda_{i}^{j} \circ s_{i}^{1}, \lambda_{i}^{j} \circ s_{i}^{2}\right]_{E_{j}}$ y por lo tanto:

$\lambda_{i}^{j} \circ\left[s_{i}^{1}, s_{i}^{2}\right]_{E_{i}}=\left[\lambda_{i}^{j} \circ s_{i}^{1}, \lambda_{i}^{j} \circ s_{i}^{2}\right]_{E_{j}}$.

Utilizando $\lambda_{i}^{j} \circ s_{i}^{a}=s_{j}^{a} \circ \varepsilon_{i}^{j}$, tenemos: $\lambda_{i}^{j} \circ\left[s_{i}^{1}, s_{i}^{2}\right]_{E_{i}}=\left[s_{j}^{1}, s_{j}^{2}\right]_{E_{j}} \circ \varepsilon_{i}^{j}$.

b) Con el fin de probar (3) vamos a establecer

$$
\begin{aligned}
\lambda_{i}^{j} & \circ\left(g_{i} \times\left[s_{i}^{1}, s_{i}^{2}\right]_{E_{i}}+\left(\rho_{i}\left(s_{i}^{1}\right)\right)\left(g_{i}\right) \times s_{i}^{2}\right) \\
& =\left(g_{j} \times\left[s_{j}^{1}, s_{j}^{2}\right]_{E_{j}}+\left(\rho_{j}\left(s_{j}^{1}\right)\right)\left(g_{j}\right) \times s_{j}^{2}\right) \circ \varepsilon_{i}^{j}
\end{aligned}
$$

porque, para $k \in\{i, j\}$, tenemos:

$$
\left[s_{k}^{1}, g_{k} \times s_{k}^{2}\right]_{E_{k}}=g_{k} \times\left[s_{k}^{1}, s_{k}^{2}\right]_{E_{k}}+\left(\rho_{k}\left(s_{k}^{1}\right)\right)\left(g_{k}\right) \times s_{k}^{2} .
$$

Podemos escribir:

$$
\begin{aligned}
& \lambda_{i}^{j} \circ\left(g_{i} \times\left[s_{i}^{1}, s_{i}^{2}\right]_{E_{i}}+\left(\rho_{i}\left(s_{i}^{1}\right)\right)\left(g_{i}\right) \times s_{i}^{2}\right) \\
& =\lambda_{i}^{j} \circ\left(g_{i} \times\left[s_{i}^{1}, s_{i}^{2}\right]_{E_{i}}\right)+\lambda_{i}^{j} \circ\left(\left(\rho_{i}\left(s_{i}^{1}\right)\right)\left(g_{i}\right) \times s_{i}^{2}\right) \\
& =g_{i} \times\left(\lambda_{i}^{j} \circ\left[s_{i}^{1}, s_{i}^{2}\right]_{E_{i}}\right)+\lambda_{i}^{j}\left(X_{i}^{1}\left(g_{i}\right)\right) \times \lambda_{i}^{j} \circ s_{i}^{2} \quad\left(\lambda_{i}^{j} \text { es un morfismo }\right) \\
& =g_{i} \times\left(\left[s_{j}^{1}, s_{j}^{2}\right]_{E_{j}} \circ \varepsilon_{i}^{j}\right)+X_{j}^{1}\left(g_{j}\right) \circ \varepsilon_{i}^{j} \times s_{j}^{2} \circ \varepsilon_{i}^{j} \quad \text { cf. (2) } \\
& =\left(g_{j} \circ \varepsilon_{i}^{j}\right) \times\left(\left[s_{j}^{1}, s_{j}^{2}\right]_{E_{j}} \circ \varepsilon_{i}^{j}\right)+\left(X_{j}^{1}\left(g_{j}\right) \times s_{j}^{2}\right) \circ \varepsilon_{i}^{j} \\
& =\left(g_{j} \times\left[s_{j}^{1}, s_{j}^{2}\right]_{E_{j}}\right) \circ \varepsilon_{i}^{j}+\left(\rho_{j}\left(s_{j}^{1}\right)\left(g_{j}\right) \times s_{j}^{2}\right) \circ \varepsilon_{i}^{j} \\
& =\left(g_{j} \times\left[s_{j}^{1}, s_{j}^{2}\right]_{E_{j}}+\left(\rho_{j}\left(s_{j}^{1}\right)\right)\left(g_{j}\right) \times s_{j}^{2}\right) \circ \varepsilon_{i}^{j} .
\end{aligned}
$$


3. Con la construcción de $\underset{\lim }{\longrightarrow}[,]_{i}$, si $\rho_{i}$ es un morfismo de algebroides $\left(E_{i}, \pi_{i}, M_{i}, \rho_{i},[,]_{i}\right) \rightarrow\left(T \overrightarrow{M_{i}, M_{i}},[],\right)$, entonces tenemos

$$
\underset{\lim }{\longrightarrow} \rho_{i}\left(\varliminf_{\longrightarrow}[,]_{i}\right)=\left[\lim _{\longrightarrow} \rho_{i}(.), \lim _{\longrightarrow} \rho_{i}(.)\right] .
$$

Es fácil probar que si cada corchete $[,]_{i}$ satisface la identidad de Jacobi, el límite $\underset{\longrightarrow}{\lim }[,]_{i}$ satisface también la identidad de Jacobi.

Sea $\left(E_{i}, \tau_{i}, M_{i}, \rho_{i}\right)_{i \in \mathbb{N}^{*}}$ una sucesión directa de algebroides de Lie donde $\left(\left(E_{i}, \lambda_{i}^{j}\right)\right)_{i \in \mathbb{N}^{*}, j \in \mathbb{N}^{*}, i \leq j}$ es la sucesión directa de fibrados asociada. Sea $\left(P_{i}, \nu_{i}, M_{i}\right)_{i \in \mathbb{N}^{*}}$ una sucesión directa de fibrados vectoriales y $\theta_{i}^{j}: P_{i} \longrightarrow P_{j}$ una aplicación fibrada sobre $\varepsilon_{i}^{j}: M_{i} \longrightarrow M_{j}$. Puesto que $\lambda_{i}^{j}$ es un morfismo entre los algebroides de Lie $\left(E_{i}, \tau_{i}, M_{i}, \rho_{i}\right)$ y $\left(E_{j}, \tau_{j}, M_{j}, \rho_{j}\right)$, entonces $T^{\lambda_{i}^{j}} \theta_{i}^{j}: \mathcal{T}^{E_{i}} P_{i} \longrightarrow \mathcal{T}^{E_{j}} P_{j}$ es un morfismo de prolongaciones de algebroides de Lie (cf. Proposición 19). Con la condición de compatibilidad

$$
\rho_{P_{j}} \circ T^{\lambda_{i}^{j}} \theta_{i}^{j}=T \theta_{\iota}^{j} \circ \rho_{P_{i}}
$$

$\left(T^{E_{i}} P_{i}, \tau_{P_{i}}^{E_{i}}, P_{i}, \rho_{P_{i}}\right)_{i \in \mathbb{N}^{*}}$ es una sucesión directa de algebroides de Lie.

Utilizando el Teorema 21 obtenemos el resultado siguiente.

Teorema $22\left(\stackrel{\lim T^{E_{i}}}{P_{i}}, \underset{\lim }{\longrightarrow} \tau_{P_{i}}^{E_{i}}, \lim _{\longrightarrow} P_{i}, \lim _{\longrightarrow} \rho_{P_{i}}\right)$ es un algebroide de Lie conveniente.

Ejemplo 23 El oscilador armónico conveniente.- Consideramos el caso del oscilador armónico que es un sistema bihamiltoniano. Consideramos aquí el límite directo de prolongaciones de algebroides de Lie $T^{E_{n}} E_{n}^{*}$ donde $E_{n}=T \mathbb{R}^{n}$ y el ancla es el tensor de Nijenhuis

$$
N_{n}=\left(\begin{array}{ccccc}
\frac{\left(x^{1}\right)^{2}+\left(y^{1}\right)^{2}}{2} & 0 & & & \\
0 & \frac{\left(x^{1}\right)^{2}+\left(y^{1}\right)^{2}}{2} & & & \\
& & \ddots & & \\
& & & \frac{\left(x^{n}\right)^{2}+\left(y^{n}\right)^{2}}{2} & 0 \\
& & & 0 & \frac{\left(x^{n}\right)^{2}+\left(y^{n}\right)^{2}}{2}
\end{array}\right) .
$$


Tenemos los corchetes siguientes: $\left[\frac{\partial}{\partial x^{k}}, \frac{\partial}{\partial y^{k}}\right]_{N_{n}}=-y^{k} \frac{\partial}{\partial x^{k}}+x^{k} \frac{\partial}{\partial y^{k}}$.

Si $\left(x^{i}, \mu_{\alpha}\right)_{1 \leq i \leq n, 1 \leq \alpha \leq n}$ son coordenadas sobre $T^{*} \mathbb{R}^{n}$ consideramos el hamiltoniano, definido para $\left(x^{i}, \mu_{\alpha}\right) \neq(0,0)$, por

$$
H_{n}:\left(x^{i}, \mu_{\alpha}\right) \mapsto \prod_{i=1}^{n} \ln \left(\left(x^{i}\right)^{2}+\left(\mu_{\alpha}\right)^{2}\right) .
$$

Tenemos una sucesión proyectiva de funciones.

Obtenemos las ecuaciones de Hamilton sobre cada $T^{*} \mathbb{R}^{n}$ (cf. [16] ):

$$
\left\{\begin{aligned}
\frac{d x^{i}}{d t} & =\rho_{\alpha}^{i} \frac{\partial H_{n}}{\partial \mu_{\alpha}} \\
\frac{d \mu_{\alpha}}{d t} & =-\rho_{\alpha}^{i} \frac{\partial H_{n}}{\partial x^{i}}-\mu_{\gamma} C_{\alpha \beta}^{\gamma} \frac{\partial H_{n}}{\partial \mu_{\beta}} .
\end{aligned}\right.
$$

Estas ecuaciones se pueden simplificar de forma que:

$$
\left\{\begin{aligned}
\frac{d x^{i}}{d t} & =\mu^{i} \\
\frac{d \mu^{\alpha}}{d t} & =-x^{\alpha}
\end{aligned}\right.
$$

\section{Referencias}

[1] Bourbaki, N. (2006) Eléments de Mathématiques, Algèbre, Chapitres 1 à $3,2^{\text {ème }}$ édition. Springer, Berlin.

[2] Cabau, P. (2012) "Strong projective limit of Banach Lie algebroids", Portugal. Math. (N.S.) 69(1): 1-21.

[3] Cabau, P.; Pelletier, F. (2012) "Almost Lie structures on an anchored Banach bundle", Journal of Geometry and Physics 62(11): 2147-2169.

[4] De las Nieves Sosa Martín, D. (2008) Algebroides de Lie y Mecánica Geométrica. Tesis, Universidad de La Laguna.

[5] De León, M.; Marrero, J.C.; Martínez, E. (2005) "Lagrangian submanifolds and dynamics on Lie algebroids", J. Phys. A: Math. Gen. 38 (24): R241-R308. 
[6] Glöckner, H. (2003) "Direct limit of Lie groups and manifolds", J. Math. Kyoto Univ. (JMKYAZ) 43(1): 1-26.

[7] Glöckner, H. (2005) "Fundamentals of direct limit Lie theory", Compositio Math. 141(6): 1551-1577

[8] Glöckner, H. (2007) "Direct limits of infinite-dimensional Lie groups compared to direct limits in related categories", Journal of Functional Analysis 245(1): 19-61.

[9] Hansen, V.L. (1971) "Some theorems on direct limit of expanding sequences of manifolds", Math. Scand. 29(1): 5-36.

[10] Higgins, P.J.; Mackenzie, K.C.H. (1990) "Algebraic constructions in the category of Lie algebroids", J. Algebra 129(1): 194-230.

[11] Iglesias Ponte, D. (2011) "Variedades de Poisson, grupoides y algebroides de Lie", Actas del XI Congreso Dr. Antonio A.R. Monteiro: 35-59.

[12] Kriegel, A.; Michor, P.W. (1997) The convenient Setting of Global Analysis. AMS Mathematical Surveys and Monographs 53, Providence RI.

[13] Lang, S. (1995) Differential and Riemannian Manifolds, Graduate Texts in Mathematics, 160. Springer, New York.

[14] Magri, F.; Morosi, C. (2008) "A geometrical characterization of integrable Hamiltonian systems through the theory of Poisson-Nijenhuis manifolds", Quaderno S 19, Università degli Studi di Milano.

[15] Martínez, E. (2005) "Classical field theory on Lie algebroids: multisymplectic formalism”, J. Phys. A: Math. Gen. 38: 7145-7160.

[16] Martínez, E. (2007) "Lie algebroids in classical mechanics and optimal control", Symmetry, Integrability and Geometry: Methods and Applications SIGMA 3, 050: 1-17.

[17] Pradines, J. (1966) “Théorie de Lie pour les groupoïdes différentiables. Relations entre propriétés locales et globales", C.R. Acad. Sci. Paris 263(25): 907-910.

[18] Suri, A.; Cabau, P. (2014) "Geometric structure for the tangent bundle of direct limit manifolds", Differential Geometry - Dynamical Systems 16: 239-247. 
[19] Weinstein, A. (1996) "Lagrangian mechanics and groupoids", Fields Inst. Comm. 7: 207-231. 